\title{
Dynamic Analysis for a Geared Turbofan Engine with Variable Area Fan Nozzle
}

\author{
Jeffrey T. Csank ${ }^{1}$ \\ NASA Glenn Research Center, Cleveland, OH, 44135, USA \\ and \\ George L. Thomas ${ }^{2}$ \\ $N \& R$ Engineering and Management Services, Inc., Parma Heights, OH, 44130, USA
}

\begin{abstract}
Aggressive design goals have been set for future aero-propulsion systems with regards to fuel economy, noise, and emissions. To meet these challenging goals, advanced propulsion concepts are being explored and current operating margins are being re-evaluated to find additional concessions that can be made. One advanced propulsion concept being evaluated is a geared turbofan with a variable area fan nozzle (VAFN), developed by NASA. This engine features a small core, a fan driven by the low pressure turbine through a reduction gearbox, and a shape memory alloy (SMA) actuated VAFN. The VAFN is designed to allow both a small exit area for efficient operation at cruise, while being able to open wider at high power conditions to reduce backpressure on the fan and ensure a safe level of stall margin is maintained. The VAFN is actuated via a SMA-based system instead of a conventional system to decrease overall weight of the system, however, SMA-based actuators respond relatively slowly, which introduces dynamic issues that are investigated in this work. This paper describes both a control system designed specifically for issues associated with SMAs, and dynamic analysis of the geared turbofan VAFN with the SMA actuators. Also, some future recommendations are provided for this type of propulsion system.
\end{abstract}

$\begin{array}{ll}\text { Ath } & \text { Nozzle throat area } \\ \text { FAR } & \text { Fuel-to-air ratio } \\ F n & \text { Thrust, lbf } \\ F n R & \text { Corrected thrust, lbf } \\ \text { GTF } & \text { Geared turbofan } \\ \text { GE } & \text { General Electric } \\ \text { HPC } & \text { High-pressure compressor } \\ \text { HPT } & \text { High-pressure turbine } \\ \text { IWP } & \text { Integral windup protection } \\ \text { LPC } & \text { Low-pressure compressor } \\ \text { LPT } & \text { Low-pressure turbine } \\ N f & \text { Fan speed,rpm } \\ N f R & \text { Corrected fan speed, rpm } \\ N h & \text { High pressure shaft speed, rpm } \\ N l & \text { Low pressure shaft speed, rpm } \\ N l R & \text { Corrected low pressure shaft speed, rpm } \\ \text { NPSS } & \text { Numerical propulsion system simulation } \\ \text { N+3 } & 3^{\text {rd generation technology from current generation }} \\ \text { PI } & \text { Proportional-Integral } \\ P s 3 & \text { Static pressure at station 3 (high pressure compressor exit), psi }\end{array}$

\footnotetext{
${ }^{1}$ Research Engineer, Power Management and Distribution Branch, jeffrey.t.csank@nasa.gov, AIAA Sr. Member

${ }^{2}$ Controls Engineer, Intelligent Control and Autonomy Branch, george.1.thomas@nasa.gov
} 


$\begin{array}{ll}\text { SLS } & \text { Sea level static (0 feet altitude and } 0.0 \text { Mach number) } \\ \text { SM } & \text { Surge margin, \% } \\ \text { SMA } & \text { Shape memory alloy } \\ \text { TSFC } & \text { Thrust specific fuel consumption } \\ T 40 & \text { Temperature at station } 4 \text { (high pressure turbine inlet), }{ }^{\circ} \mathrm{R} \\ \text { VAFN } & \text { Variable area fan nozzle } \\ W f & \text { Fuel flow, lbm/s } \\ W f / P s 3 & \text { Control limiter, fuel flow divided by high pressure combustor static pressure }\end{array}$

\section{Introduction}

T HE National Aeronautics and Space Administration (NASA) is investing in the future of commercial air travel by focusing on advanced aircraft concepts. These future concepts are expected to enter into service in the 20302035 time frame and are known as N+3 technologies; these being concepts and technologies targeted for third generation aircraft beyond the current generation. The $\mathrm{N}+3$ strategy contains aggressive performance and environmental metrics including a $71 \mathrm{~dB}$ reduction in noise, $75 \%$ reduction in nitrogen oxides $\left(\mathrm{NO}_{\mathrm{x}}\right)$ emitted during landing and takeoff, and $70 \%$ reduction in aircraft fuel burn relative to the state of the art reference aircraft (Boeing 737-800 aircraft with CFM56-7B engine). ${ }^{1,2}$ There are a variety of proposed future propulsion concepts belonging to various classes. One such class is a parallel hybrid-electric, which includes the Boeing-General Electric (GE) "Sugar Volt" engine that features a conventional turbofan with an integrated motor connected to the low pressure shaft. ${ }^{3}$ Another class is a distributed hybrid-electric, such as the NASA N3-X that features distributed motor-driven fan propulsors integrated within a hybrid wing body airframe. ${ }^{4}$ Yet another class is turbo-electric, such as the STARC-ABL that features a tail-cone mounted electric ducted fan for boundary layer ingestion, driven by power takeoff from two conventional underwing turbofans. ${ }^{5}$ Finally, there are advanced, non-hybrid gas turbine propulsion systems. These systems may include ultra-high engine bypass ratios, reduction gearbox-driven fans, or additional effectors such as a variable area bypass duct nozzle, referred to in this paper as a variable area fan nozzle (VAFN). ${ }^{6}$ The propulsion system investigated in this work is a geared turbofan with a VAFN. ${ }^{2}$

The VAFN is an effector that allows the exit area of the nozzle at the end of the engine bypass duct to be adjusted for improved performance. The VAFN design is being investigated for its ability to reduce noise and fuel burn. ${ }^{7,8}$ Current commercial turbofan aircraft engines are designed and optimized at the design point, typically cruise, and simulated and analyzed at other critical points in the mission profile, such as takeoff and climb, to ensure the design meets the design constraints (steady-state) throughout the profile. In a traditional engine configuration, the fan nozzle area is fixed and must provide satisfactory performance throughout the mission. It is noted that decreasing the area of the fan nozzle generally improves efficiency at cruise; however, the fan nozzle area that is optimal for cruise could cause the engine to surge at other flight conditions. ${ }^{7}$ With a VAFN, the nozzle area can be adjusted between takeoff and cruise, allowing for improved performance at cruise while providing the additional surge margin at takeoff by allowing a larger nozzle area at other flight conditions. It has also been reported in Ref. 8 that the VAFN can reduce fuel burn by $2 \%$ at cruise and $10 \%$ during departure and approach while reducing cruise noise by $1.0 \mathrm{~dB}$. A more detailed list of advantages associated with the VAFN can be found in Ref. 8.

Traditionally, variable area nozzles have only been used for military and afterburning engines. This is because military aircraft often prioritize performance, and can afford the additional weight and complexity that typically comes with effectors like variable area nozzles. Further, in afterburning engines, the engine mass airflow is dramatically higher in wet versus dry operation, and very different nozzle areas are needed for proper matching for these different operating modes. ${ }^{9}$ However, the weight and complexity associated with traditional electrical or hydraulic actuators that have been used with variable area nozzles ${ }^{9}$ makes them difficult to justify for commercial aeropropulsion applications. Shape memory alloy (SMA) actuators have been proposed as an alternative due to their high power-toweight ratio and simplified solid-state actuation. ${ }^{10}$ SMAs are made of an alloy that can change shapes based on temperature; this temperature dependent, shape-changing phenomenon is known as the shape-memory effect.

Reference 10 describes a prototype variable area nozzle consisting of eight overlapping leaves or petals that are controlled by four SMA wire actuators. These actuators are located away from the exhaust nozzle to avoid the extremely high temperatures. The SMA wires contract when heated, and these contracting forces are transmitted via wires, pulleys, and connecting rods to a sliding ring. Springs are used to apply pretension for the SMA elements and provide returning forces. The sliding ring is designed to move all of the nozzle area petals simultaneously, and it is the displacement of the sliding ring that is controlled to achieve the desired nozzle area. During open-loop testing, it was observed that it took the SMA actuator $9.8 \mathrm{~s}$ to reach full stroke (minimum area), achieved by heating the shape memory alloy. It also took $25 \mathrm{~s}$ for the SMA to return to its original position, via heat dissipation, which could be

American Institute of Aeronautics and Astronautics 
improved by using forced convection. A nozzle area decrease of over $40 \%$ was observed, for nominal to full stroke actuator displacement. ${ }^{10}$ In addition to the slower response times of SMA actuators, other challenges include a temperature dependent hysteresis and potential shape memory loss when exceeding temperature limits. ${ }^{11}$ Note that instead of expanding the SMA by heating and contracting it by cooling, the SMA actuators can also be configured as arrays of elements that oppose each other to achieve similar actuation with approximately the same response times. ${ }^{7}$

This paper discusses the preliminary dynamic analysis of the geared turbofan propulsion system with a variable area fan nozzle, focusing on analyzing the relationship between the dynamic response and operability (stability) margins. Section II contains details regarding the engine model. Background information about the dynamic systems analysis process is given in Section III. Details of the control system are found in Section IV followed by the dynamic analysis of the NASA geared turbofan in Section V. Section VI offers some concluding remarks.

\section{NASA Geared Turbofan Variable Area Fan Nozzle Engine Model}

The NASA geared turbofan (GTF) with a VAFN engine model was designed using the Numerical Propulsion System Simulation (NPSS) ${ }^{12}$, and is configured to work with MATLAB ${ }^{\circledR}$ via an S-function. ${ }^{13}$ The NPSS S-function allows the actual NPSS engine model to be executed each time step, via Simulink, and provide the results back to Simulink. The GTF with VAFN model contains a fan component connected to the low pressure shaft via a gearbox designed to reduce the speed of the fan (Nf). The low pressure compressor (LPC) and turbine (LPT) are connected by the low pressure shaft. The high pressure compressor (HPC) and high pressure turbine (HPT) are connected via the high pressure shaft. The speeds of the low pressure and high pressure shafts are denoted $N l$ and $N h$ respectively. The fan, compressors, and turbines are modeled using performance maps that relate the pressure ratio, mass flow rate, corrected speed, and thermodynamic efficiency for each component. In this configuration, the bypass flow and core flow are split after the fan component. The bypass flow exits from the bypass nozzle, or VAFN, where the area is affected by the shape memory alloy actuator.

The nozzle area primarily impacts the operation of the components in its flow path. For example, changing the bypass nozzle area mainly impacts the fan operating line, or op-line. The op-line is the pressure ratio and corrected flow relationship that the engine will operate at in steady-state. Previous VAFN designs vary the bypass nozzle area based on flight condition (especially Mach number), where the area is larger at take-off and initial climb and is smaller at cruise. ${ }^{7,8}$ This method implies that the fan map operating lines are defined based on the flight condition, or that a fixed nozzle area is defined for a given flight condition. For example, the bypass nozzle area and fan op-line could be defined for take-off, climb, cruise, approach, and landing. The control system then would be designed to transition the SMA-based VAFN between these target design areas while the engine is transitioning between flight conditions.

The NASA VAFN engine concept does not follow the same approach. The VAFN area is designed to allow the fan to operate on a constant op-line during steady-state operation. The compressor (and fan) maps used in this work are parameterized by corrected speed and r-line, which together, create uniquely defined coordinate systems on compressor maps. R-line is an arbitrarily chosen auxiliary parameter that may or may not correlate to a physical parameter (such as specific work) ${ }^{14}$ An r-line of 2.0 is chosen as the desired op-line because the desired cruise point on the fan map exists at $r$-line $=2.0$; this op-line is shown on the fan map in Figure 1 as the heavy solid blue line. Achieving this op-line requires the VAFN area to be a function of the power level, since, given a constant corrected speed, a unique bypass nozzle area can be chosen to force the fan to operate on a desired op-line. For this application, the corrected low pressure shaft speed (NlR, $R$ indicating a corrected parameter ${ }^{15}$ ) is chosen as the parameter by which the nozzle area is scheduled. This is because the speed parameter on the fan map (corrected fan speed $(N f R)$ ) is not directly measured. As an alternative to corrected fan speed, $N I R$ can be used in its place for the nozzle schedule, because $N I R$ is approximately equal to $N f R$ times the gear ratio. The control schedule for the VAFN as a function of $N I R$ is shown in Figure 2; this schedule is defined over a range of about 2500 to $6000 \mathrm{rpm}$, as this is the speed range from idle to full power.

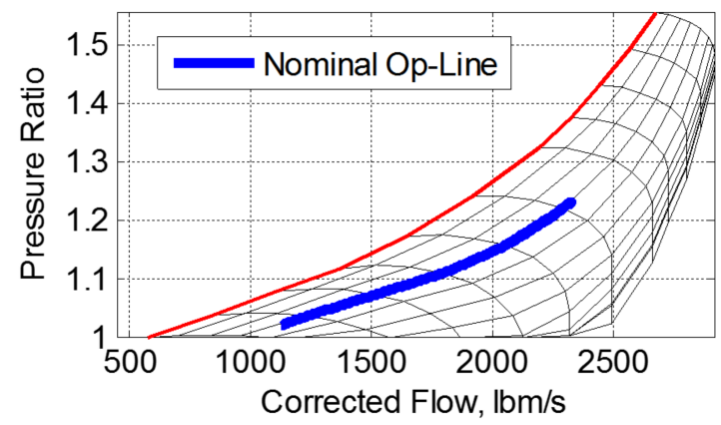

Figure 1. Fan map showing the steady-state operating line at a constant $r$-line of 2.0.

American Institute of Aeronautics and Astronautics 


\section{Dynamic Systems Analysis}

Previous dynamic systems analysis (DSA) studies ${ }^{16,17}$ analyzed the relationship between the dynamic performance (acceleration response time) and operability margin (minimum HPC surge margin) for an idle to takeoff throttle transient at sea level static (SLS) conditions, defined as an altitude of 0 feet and Mach 0.0. These studies used transient simulation data to identify this relationship for particular engine designs. Given this performanceoperability relationship, recommendations were made regarding the steady-state design constraints. Note that these analyses focused on analysis of minimum HPC surge margin and acceleration response time because these variables show a negative correlation for most engines. This is in contrast to minimum LPC and fan surge margin and most other operability variables, which are not as strongly correlated to acceleration response time, and may instead be correlated to deceleration response time (an unconstrained variable).

The steady-state HPC surge margin constraint represents a safety margin between HPC surge and normal operating conditions; this constraint includes allowances for several off-nominal phenomena, including a variety of kinds of modeling uncertainty (known as the uncertainty stack) and transients (known as the transient stack). The items that factor into in the uncertainty stack include changes in engine performance as components degrade over an engine's lifespan, ambient condition uncertainties such as inlet distortion, engine-to-engine variation, as well as others. ${ }^{18}$

The uncertainty stack is computed as a sum of the worst case surge margin debits that can be caused by all of the uncertainties accounted for. For the purposes of control design, this number is the minimum surge margin that the controller can allow in the closed-loop system during transients and still avoid surge in all uncertainty conditions. The steady-state surge margin constraint is the sum of the uncertainty stack surge margin value and the transient stack value. The transient stack represents the worst case surge margin debit that is expected to occur when transitioning from one flight condition or engine power state to another.

Figure 3 shows a notional compressor map and its operating line (op-line), which is the steady-state trajectory that the compressor operating point follows on its map as it changes speed or power level (shown as a solid red line) when integrated into an engine system. During an acceleration transient, the HPC typically leaves the op-line, moves toward the surge line, and then settles back onto the op-line as the transient settles out (this transient trajectory is shown as the red dotted line in Figure 3). The green dashed line in the figure represents a unique line on the compressor map with a constant surge margin equal to the uncertainty stack value. This represents a line that the control logic must keep the compressor from crossing by limiting how fast transients are performed.

Because the location of the op-line on the compressor map (and thus the steady-state surge margin) is consequence of the engine system design process, the surge margin constraint (the sum of the transient and uncertainty stacks) is a value that is built into the engine during the design phase.

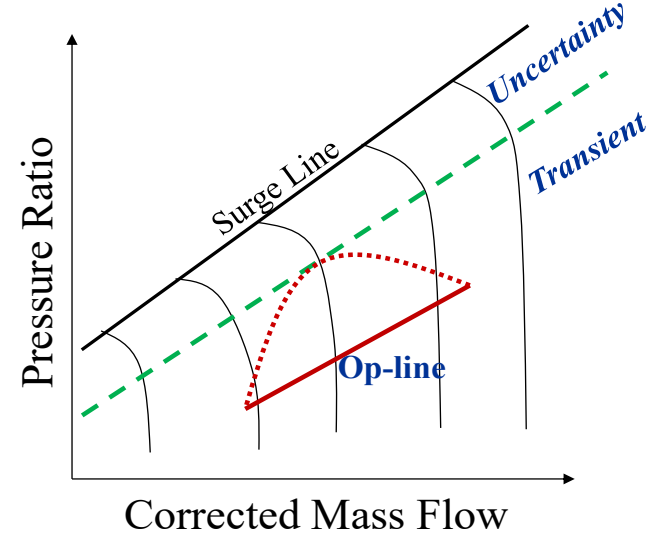

Figure 3. Generic compressor map showing the uncertainty stack, transient stack, op-line (solid), and path during transient operation (dotted). Since engine designs that run the HPC closer to surge typically exhibit better fuel efficiency, it is advantageous to design an engine with an appropriately sized transient surge margin stack, but no bigger. This motivates the development of tools and methods to precisely quantify the necessary transient stack for a given engine design.

Note that, as the control logic is redesigned to execute acceleration transients more slowly, the resulting minimum HPC surge margins typically increase. This means that proper control design can reduce the necessary size of the

American Institute of Aeronautics and Astronautics 
transient stack by slowing the rate of accelerations. However, also note that there are performance constraints that the closed-loop engine system must satisfy, most notably for maximum acceleration response time. The U.S. Federal Aviation Adminstration (FAA) Regulation, Part 33, Section 33.73, requires that engines must be able to spool up from less than $15 \%$ of their rated takeoff thrust to greater than $95 \%$ of their rated takeoff thrust in not over 5 seconds when tested at a static condition. ${ }^{19}$ This is referred to later in this paper as the "5 second requirement."

The appropriate size of the transient stack is quantified in the DSA process. This process involves designing a family of controllers that protect different minimum HPC surge margin design values, and simulates the engine with these controller designs to obtain the acceleration response time and minimum HPC surge margin. The trend in the response times and minimum surge margins obtained from the different controller designs highlights the possible tradeoffs between performance and reliability that are possible for a given engine design.

Figure 4 shows three notional control design solutions for a given engine, each achieving a different tradeoff between response time and HPC surge margin. Observing the trend in the data, if a controller were designed that runs the engine fast enough so that the minimum HPC surge margin just meets the operability constraint (the uncertainty stack value, shown with a green dashed line), then that closed-loop system would use all of the available transient stack and would safely accelerate as fast as possible given the engine design. However, there is no clear benefit to accelerating an engine as fast as possible. On the other hand, if a controller was designed to just meet the performance requirement (response time, shown with a solid red line), then the closed-loop system would accelerate as fast as necessary, but no faster, and would use as little of the transient stack as possible. Observing the amount of the available transient stack surge margin used by this system during the transient will indicate exactly how much is needed. The recommendation of this DSA study would be to reduce the size of the steady-state HPC surge margin constraint such that it

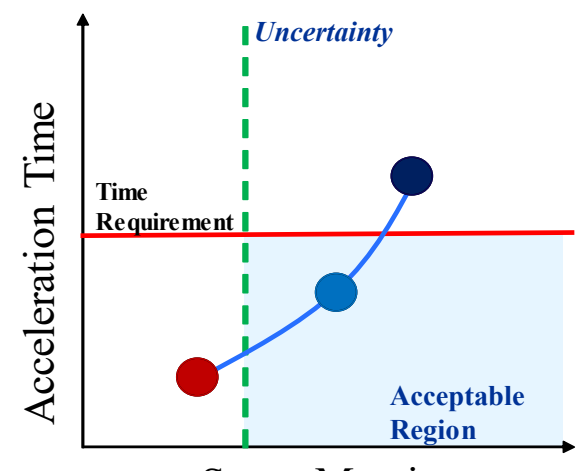

Surge Margin

\section{Figure 4. Generic plot comparing performance and operability tradeoff with the acceptable region (shaded).} includes the uncertainty stack and the smallest necessary transient stack. Reducing this constraint as recommended can open up the engine cycle design space, because it will mean that some candidate engine designs that previously violated this constraint will no longer do so.

This kind of DSA study is especially attractive because it can be performed in a transient engine simulation environment, and so it can be incorporated early in the engine design phase. However, note that in order to more accurately estimate the optimal transient stack, it is important to incorporate uncertainty analysis into the DSA process. For instance, if the performance-operability trend is assessed as shown in Figure 4 by only running a single simulation with each controller design on the nominal engine model (which typically represents a mid-life engine), then the results will likely underestimate the necessary transient stack. This is because end-of-life engines, and even newer engines with specific combinations of component degradation will tend to run with less remaining HPC surge margin (with their HPCs operating closer to the surge line). Because of this, the DSA should be conducted, keeping in mind that the objective is to find the minimal size of the transient stack such that the engine never stalls under any possible combination of uncertainty conditions and when performing any possible transient.

\section{Control Design Process}

In order to characterize and analyze the performance-operability relationship, a realistic dynamic closed-loop controller must be designed and integrated with the engine model. To aid in this process, the MATLAB/Simulinkbased Tool for Turbine Engine Closed Transient Analysis (TTECTrA) ${ }^{20}$ is used in this work to automatically tune a controller to provide the appropriate dynamic response based on user defined constraints, such as controller bandwidth, transient response requirements, and stability margins. This work uses both the TTECTrA tool and the NPSS S-function together to facilitate building closed-loop control systems around an NPSS engine simulation. ${ }^{21}$

The slow dynamic response of the SMA-based VAFN in this engine model poses additional challenges for the dynamic systems analysis process and control system design. Note that the majority of the thrust produced by a turbofan engine is attributed to the airflow through the bypass nozzle. Because the engine transient response is required to be within $5 \mathrm{~s}$, while the SMA has a much slower actuation response, the controller may not be able to exactly achieve the commanded engine thrust until the slow VAFN transient settles out and reaches its scheduled set-point

American Institute of Aeronautics and Astronautics 
value (based on operating conditions). This complicates the engine control design because it was determined in the course of this work that the VAFN achieves less than half of its commanded displacement during the required $5 \mathrm{~s}$ response time. This means that the power management controller must compensate for the slow response of the VAFN to arrive at its scheduled position.

Since thrust is not measurable in flight, a surrogate variable (also called control variable) that has an approximate linear relationship to thrust is used to indirectly control thrust. Often, either the rotor speed or engine pressure ratio is used as this surrogate variable. ${ }^{22}$ Note that the bypass nozzle area will have a large impact on the relationship between thrust and its possible surrogate variables. This means that any control logic that computes the appropriate control variable set point given a thrust demand must take into account the bypass nozzle area in order for its set point values to correctly correspond to the desired thrust. In practice, this means another dimension for the set point control lookup table will be necessary. However, this additional complexity will not be incorporated in this preliminary study.

Given the advances in distributed engine control (additional computing power) ${ }^{23}$ and model-based engine control (Kalman Filtering), ${ }^{24,25}$ it is possible that the $\mathrm{N}+3$ control system architectures will also be able to estimate thrust more accurately. Thus, this work assumes that thrust can be estimated accurately in real time, and so the controller in this work is designed to directly control the engine's thrust. This assumption is made to simplify the control design, as regulating thrust directly removes the need for additional logic to compensate for off-schedule VAFN operation. It is considered acceptable to make this simplifying assumption, because the control designs created in this work are not made for their own sake, but for the sake of analyzing the engine's transient performance in a closed-loop system.

This work uses a modified version of the original TTECTrA code. The controller architecture used in this version of TTECTrA is shown in Figure 5. The control logic elements that are designed by TTECTrA are shown in blue. The first is the proportional-integral (PI) thrust controller with integral windup protection (IWP). This block produces a fuel flow command that drives the sensed or estimated engine thrust level to a given command.

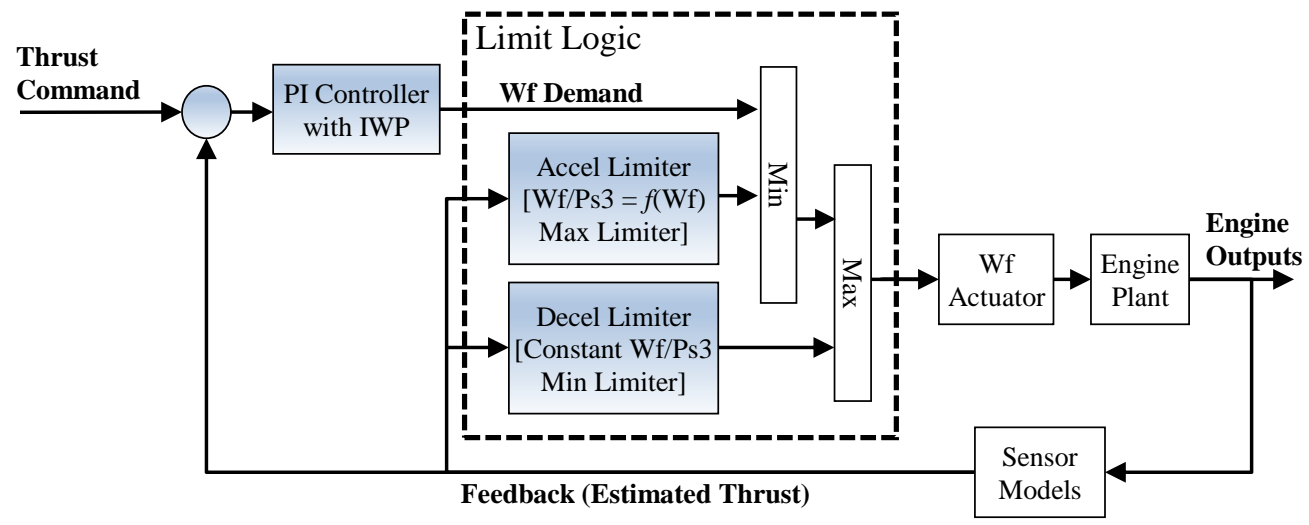

Figure 5. TTECTrA controller architecture used in this work.

The fuel flow command produced by the PI controller (Wf Demand) is limited by the acceleration (Accel) limiter block shown in Figure 5 to protect minimum HPC surge margin and maximum HPT inlet temperature (T40) during accelerations. Further, the deceleration (Decel) limiter block limits fuel flow to protect minimum LPC surge margin and the minimum fuel-to-air ratio (FAR) during decelerations. The Accel limiter produces a maximum fuel flow which limits the maximum ratio of fuel flow ( $W f)$ to combustor static pressure $(P s 3)$, or $W f / P s 3$. The maximum value of $W f / P s 3$ is based on the current $W f$ commanded to the fuel flow actuator. The Decel limiter is based on a fixed minimum $W f / P s 3$ value. The Wf/Ps3-based Accel limiter replaces the core acceleration-based limiter in the original version of TTECTrA. This new Accel limiter is simpler to integrate and tune automatically since it does not contain an internal PI regulator. The PI regulator and its Integrator Wind-up Protection (IWP) logic gains all require tuning, which increases the complexity of automatic design of controllers using the TTECTrA tool.

With the controller architecture chosen, and the engine model integrated with the TTECTrA tool, the next step is to use TTECTrA to automatically design the closed loop controller. The first step of this design process is to capture the steady-state performance of the system and use this data to design the gain scheduled set point controller (PI gains as a function $N I R$ and ambient conditions). Because this involves a steady-state characterization of the system, the VAFN dynamics are ignored for this step, meaning the VAFN area is adjusted to always operate on its intended opline (r-line of 2.0). These set point controller gains are calculated in TTECTrA using MATLAB's pidtune function based on a piecewise linear model provided by NPSS, which is a function of thrust and operating conditions. The gains are calculated for each point based on a bandwidth and phase margin requirements of 1.1 radians/second and 60 degrees, respectively.

American Institute of Aeronautics and Astronautics 
In the next step, the acceleration limiter is found using a process similar to that in the original version of TTECTrA. The limiter is designed for a maximum T40 and a minimum HPC surge margin achieved during the acceleration. The maximum Wf/Ps3 schedule is then computed by TTECTrA from data obtained by running open-loop Wf ramp-up profiles to calculate the transition time from one operating point to the other without exceeding these limits. Using a binary search process, TTECTrA finds the fastest Wf/Ps 3 acceleration curves that can be achieved without violating the transient limits when starting from several different initial power levels. The deceleration limiter is designed for an LPC surge margin and minimum fuel-to-air ratio (FAR). This open-loop $W f$ is ramped down until the engine reaches any one of its deceleration design limits (minimum FAR or LPC surge margin). The minimum Wf/Ps3 limit value is chosen to be the Wf/Ps3 value obtained when the engine reaches this design limit. In the final step, the simulation is run in closed-loop and the IWP gain is tuned to ensure that the limiters can protect the engine and still provide the necessary dynamic response.

\section{Dynamic Analysis}

The first step in the dynamic analysis process is to analyze the dynamic performance of the closed-loop control system. This engine model is rated for 29,000 lbf thrust at SLS conditions on a standard day (59 ${ }^{\circ} \mathrm{F}$ air temperature). The modified TTECTrA tool is used to automatically design the closed-loop controller, including limit logic, to meet the following steady-state and transient constraints:

- maximum HPT inlet temperature of $3400{ }^{\circ} \mathrm{R}$

- maximum FAR of 0.06

- minimum FAR of 0.012

- minimum HPC surge margin of 14\% (transient)

- minimum LPC surge margin of $10 \%$ (transient)

- $\quad$ set point controller bandwidth 1.1 radians per second.

Once the controller is designed and saved, the VAFN SMA dynamics were integrated with the engine model. Since this is a preliminary study, a simple model is used, consisting of a linear first order filter with a bandwidth of 0.09 radians per second, which has a time response of approximately 45 seconds for both opening and closing of the nozzle area. This was considered to be a conservative estimate for the response times of SMAs.

\section{A. Dynamic VAFN Analysis}

This subsection describes some of the issues associated with controlling an engine with a slow SMA-based VAFN, including challenges meeting the 5 second performance requirement. In order to highlight these issues, transient simulations with accel and decel step commands (15\% to $100 \%$, and $100 \%$ to $15 \%$ rated SLS thrust respectively) were run with the closed-loop system described in Section III, both with and without the VAFN actuator dynamics.

Figure 6 shows the net thrust, HPC surge margin (SM), and T40 during the accel and the decel portions of the simulation. This figure shows that, when running with the closed-loop controller, the transient response of these variables is substantially the same when running with or without the VAFN dynamics. This suggests that the VAFN dynamics do not significantly affect thrust, HPC surge margin, and T40.

Figure 7 shows fan and LPC surge margin, as well as the VAFN scheduled command signal and the actuator response for both tests (with and without the VAFN dynamics). This figure shows that the minimum fan surge margin is significantly lower (about 10\% absolute surge margin units) during a decel with the slow VAFN dynamics compared to without the dynamics. This is because the slow VAFN is not able to open up to its steady-state low-power area quickly enough to keep the fan from moving towards the surge line during the decel. The LPC surge margin is not significantly affected by the slow dynamics. The most significant fact shown in Figure 7 is that, as one may expect, the slow VAFN actuator causes the fan surge margin to suffer during decel transients. This occurs because a slow VAFN will not be able to quickly open up its area during the decel to relieve backpressure on the fan and reduce its surge margin.

Figure 8 shows the fuel flow, and low and high pressure shaft speeds. It indicates that, during an accel, the fuel flow and low pressure shaft speed (and to a lesser degree, high pressure shaft speed) must overshoot their final steadystate values in order to quickly reach the desired thrust setpoint with slow VAFN dynamics. The slow dynamic response causes the VAFN to not be able to completely close to its scheduled high-power area until significantly after the accel transient. This means that the VAFN will respond with a larger than commanded area during the accel, and this larger area will require the engine to run with a higher fan speed to achieve the same thrust. This illustrates a challenge associated with meeting the 5 second thrust response requirement with slow VAFN dynamics. Namely, either the VAFN must be fast enough to travel to its commanded value during a 5 second accel, so that the rated thrust can be achieved when running to the nominal max power $W f$, or otherwise, the controller must compensate and issue

American Institute of Aeronautics and Astronautics 

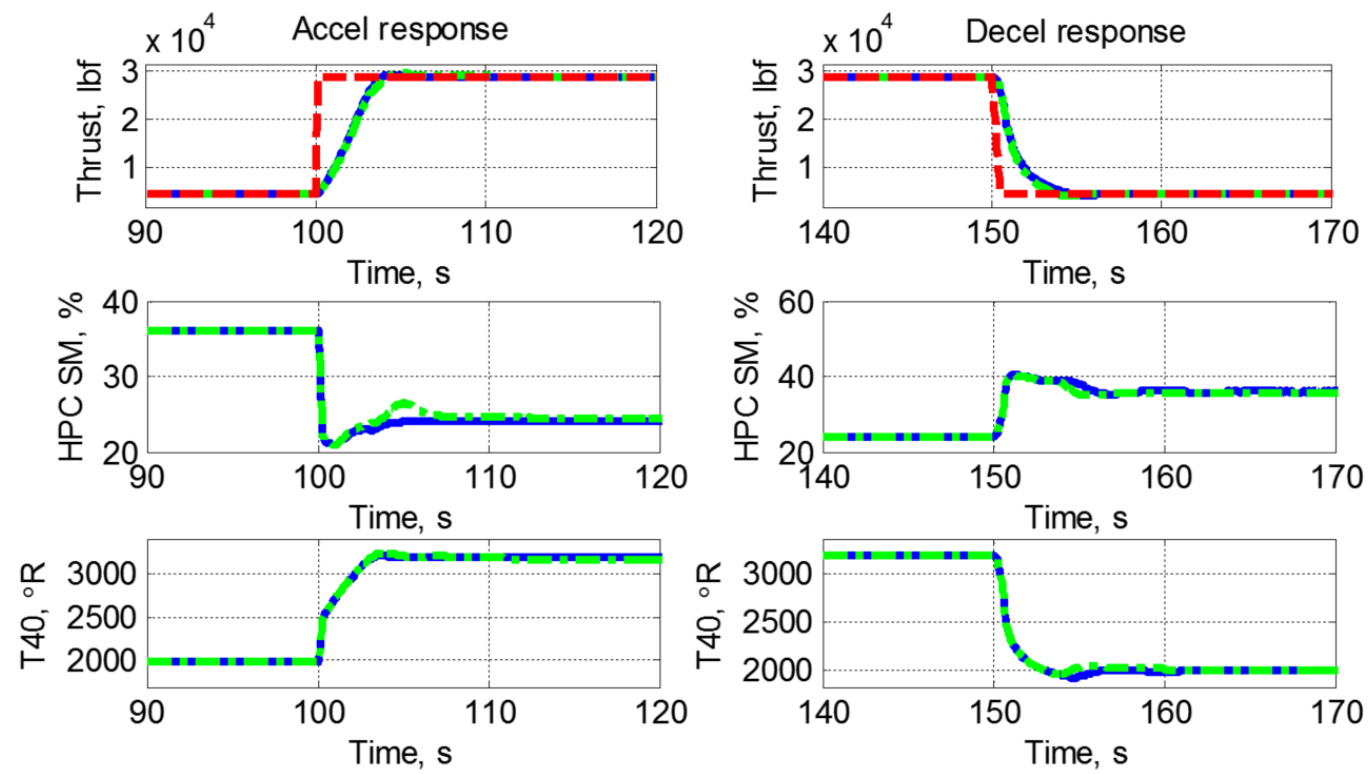

Response (No Actuator Dynamics) - = Response (Slow Actuator Dynamics) - - - Command

Figure 6. Plots showing net thrust (top), HPC surge margin (middle), and T40 (bottom) response to acceleration (left) and deceleration (right) commands, obtained by running the nominal closed-loop geared turbofan system with (green dash-dot line) and without (blue solid line) the VAFN dynamics.

a greater than nominal $W f$ command during the accel to reach the rated thrust with the larger than nominal nozzle area caused by the slow dynamics.

Further, if the controller is constructed so that it runs a higher fan speed to compensate for a larger than scheduled VAFN due to the slow actuator, care must be taken to ensure that running these larger than nominal fan speeds does
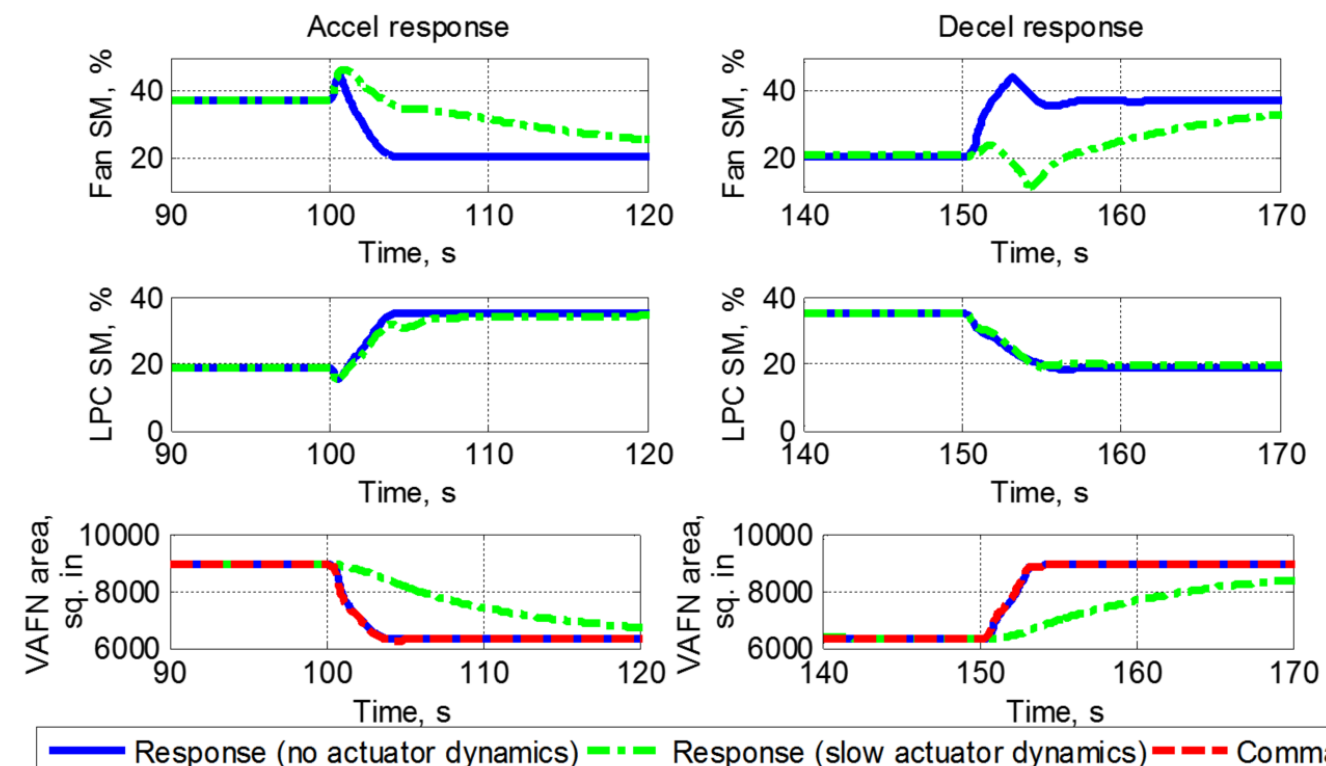

Response (no actuator dynamics) =-" Response (slow actuator dynamics) $=-=$ Command

Figure 7. Plots showing fan surge margin (top), LPC surge margin (middle), and VAFN command and response (bottom), obtained by running acceleration (left) and deceleration (right) commands on the nominal closed-loop geared turbofan system with (green dash-dot line) and without (blue solid line)

the VAFN dynamics. 

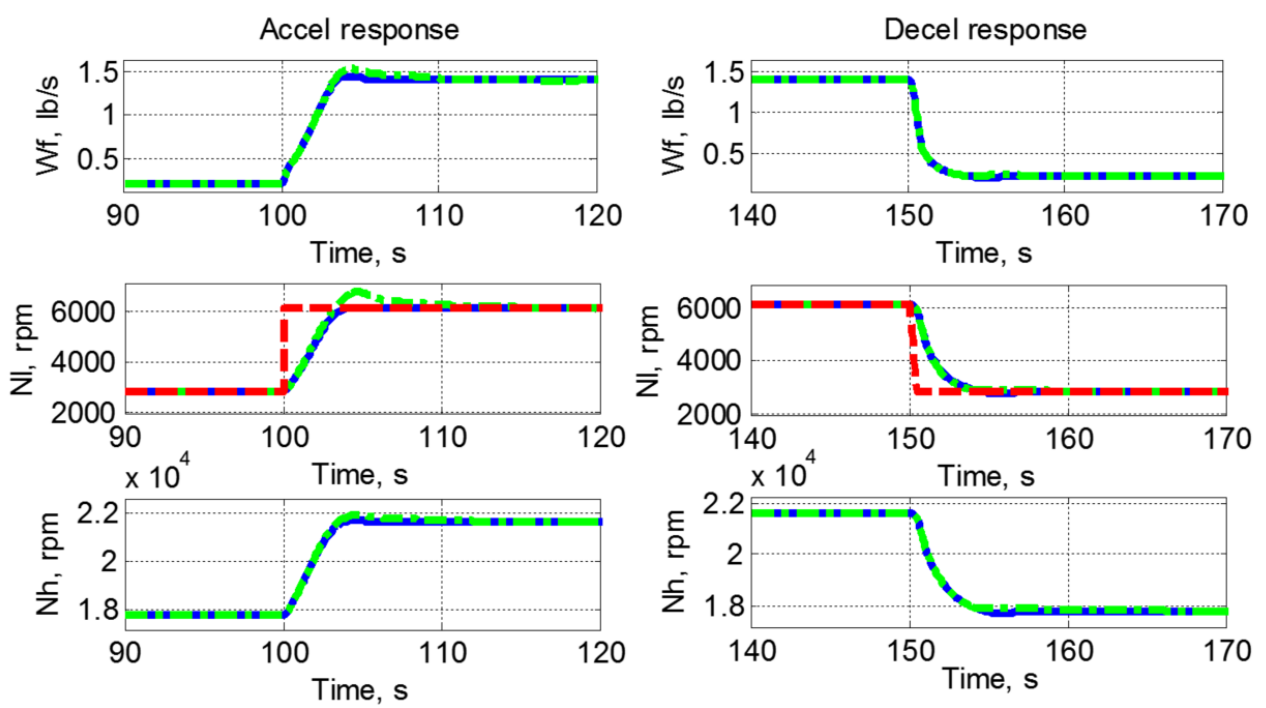

Response (no actuator dynamics) ="- Response (slow actuator dynamics)=-- Command

Figure 8. Plots showing fuel flow (top), low pressure shaft speed (middle), and high pressure shaft speed (bottom), obtained by running acceleration (left) and deceleration (right) commands on the nominal closed-loop geared turbofan system with (green dash-dot line) and without (blue solid line) the VAFN dynamics.

not mean violating maximum component speed safety limits. Note that if the slow VAFN requires the fan to run at a certain percent speed above its steady-state maximum to meet performance requirements, this may indirectly place constraints on component design, as the other components on the low pressure shaft or fan shaft must also be able to safely run at this higher than nominal speed.

Figure 9 shows how the fan, LPC, and HPC behave on their respective performance maps with the slow VAFN dynamics (dash-dotted green line) and without the VAFN dynamics (solid blue line). The trajectory of the HPC on its
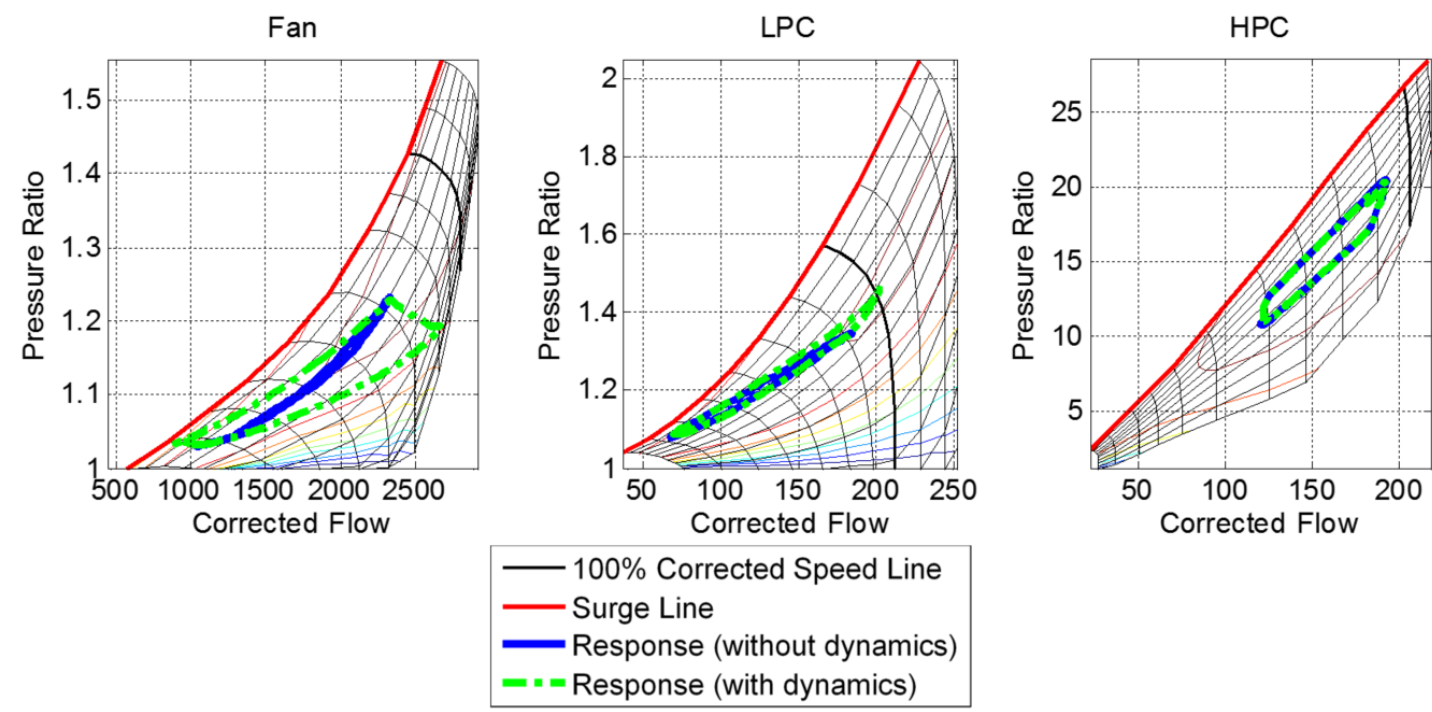

Figure 9. Plots showing the fan (left), low pressure compressor (middle), and high pressure compressor (right) performance maps for the $\mathrm{N}+3$ geared turbofan, showing the closed loop response to an accel and decel without the VAFN actuator dynamics (solid blue line), and with the slow dynamics (green dash-dot line).

American Institute of Aeronautics and Astronautics 
map is not significantly affected by the VAFN dynamics. The LPC trajectory is only affected in that the LPC runs to a higher corrected speed (the direction of the LPC map trajectory or its deviation from the op-line do not significantly change). This happens because the controller drives the fan and low pressure shafts to higher speeds to achieve the target thrust, given that the slow VAFN runs with a larger than scheduled area during the accel. These facts about the effect of the VAFN on the LPC and HPC suggest that, as expected, the VAFN does not significantly affect the core compression system. However, the slow dynamics cause the fan to travel far to the right on the map during accels, and closer to the surge line on the left during decels, whereas, with instantaneous VAFN dynamics, the fan essentially follows its steady-state op-line with a small amount of deviation during transients.

The results shown in Figure 6Figure 9 indicate that a slow VAFN will compromise fan surge margin during fast decels. This is not a significant issue for normal transient operation, because a deceleration schedule could be designed to ensure that the engine decelerates slowly enough so that the fan speed matches the transient VAFN area. This is possible because there are no deceleration performance requirements mandated by commercial aviation authorities, and no other reasons dictating that an engine be able to perform a snap decel in a given period of time. Further, in order to be able to meet the 5 second accel performance requirement with a slow VAFN, the closed-loop system must be able to safely run at a higher than steady-state fan speed until the VAFN reaches its final commanded value. This means that the engine components must be designed to operate at these maximum speeds during transients. The remainder of this paper assumes that the maximum speed values that the closed-loop GTF system runs to are not problematic.

\section{B. VAFN Analysis at Idle}

The NASA GTF reports a VAFN area of 16500 sq. in at SLS, low power ( $9 \%$ of the $29000 \mathrm{lbf}$ rated $F n$ ), and area of 6200 sq. in at SLS max power $(100 \%$ rated $F n)$. The normalized change in area is approximately $62 \%$, while other designs ${ }^{8}$ report about $25 \%$. This larger than typical change is caused by the fact that this VAFN is designed to make the fan component operate on a particular op-line $(r-l i n e=2.0)$, requiring significantly different bypass areas between low and high power operation. The VAFN can adversely affect fan surge margin at idle, because the fan back pressure increases if the nozzle area is too small when operating near idle. This increased back pressure causes an increase in pressure ratio across the fan tip, reducing the surge margin and potentially stalling the fan. For high power operation, if the nozzle area is too large, the fan chokes and the airflow saturates because the nozzle is no longer restrictive enough to generate sufficient back pressure for the fan to be in its normal operating area (between the surge and choke regions). ${ }^{18}$ The VAFN schedule must be designed to avoid these phenomena.

When including the slow SMA dynamics, the actual VAFN area lags behind its commanded value significantly during transient operation and the fan deviates from the desired op-line $(r-l i n e=2.0)$. Because of the slow actuator dynamics, the VAFN only achieves about $1 / 3$ of its travel toward the commanded value five seconds after the start of a given transient as shown in Figure 7. During engine accelerations, the off-nominal VAFN can cause the fan to venture into the choked region at the bottom right of its performance map as shown in the slow actuator fan map data in Figure 9. Note that the data shown in these figure runs the engine to $15 \%$ rated $F n$ for the low power condition part of the test. In cases where the engine is run to significantly lower power levels ( $>12 \% \mathrm{Fn}$ ), the fan runs off of the map to the right and fails to converge, because the VAFN area is too large during the acceleration transient.

In order to prevent the fan from operating near the choked region (bottom right) of the map during accelerations starting from very low power, the VAFN area at low speed was reduced. The new VAFN schedule produced by constraining the maximum VAFN area (at low speed) is shown with the original schedule in Figure 10. The opline (steady-state) obtained by running with the modified VAFN schedule is shown with a pink dotted line in Figure 11, and can be compared to the solid blue line showing the nominal op-line in the same figure. This data shows that the modified VAFN area schedule pushes the fan op-line slightly away from r-line of 2.0 and towards the surge line in the idle region (bottom left). This small shift of the op-line on the map towards surge results in a reduction of about $2 \%$ steady-state

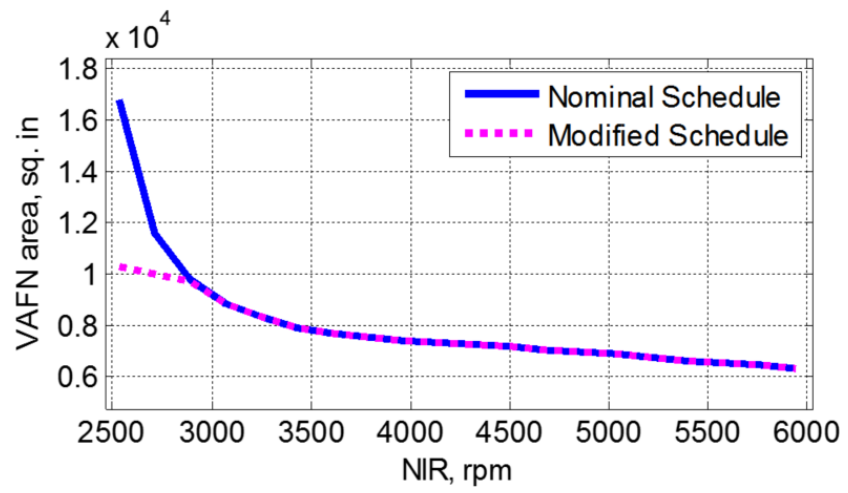

Figure 10. The nominal and modified VAFN area schedules as a function of corrected low pressure shaft speed.

American Institute of Aeronautics and Astronautics 
surge margin at a power level of $15 \%$ of the max takeoff thrust of 29,000 lbf. However, the change eliminates issues with the fan going off its map.

As shown in Figure 10, the VAFN area varies by approximately $40 \%$ in to the modified schedule, comparing the nozzle areas specified at the maximum $N I R$ value to that specified at the minimum $N l R$ value in the schedule. This range is closer to what has been demonstrated in literature than the original nozzle area variation of $62 \% .^{8}$ The modified schedule results in the VAFN starting closer to its final value during an acceleration transient. This means that the nozzle will operate closer to its nominal area during the acceleration and will thus require less additional fuel or low pressure shaft speed to reach the target thrust.

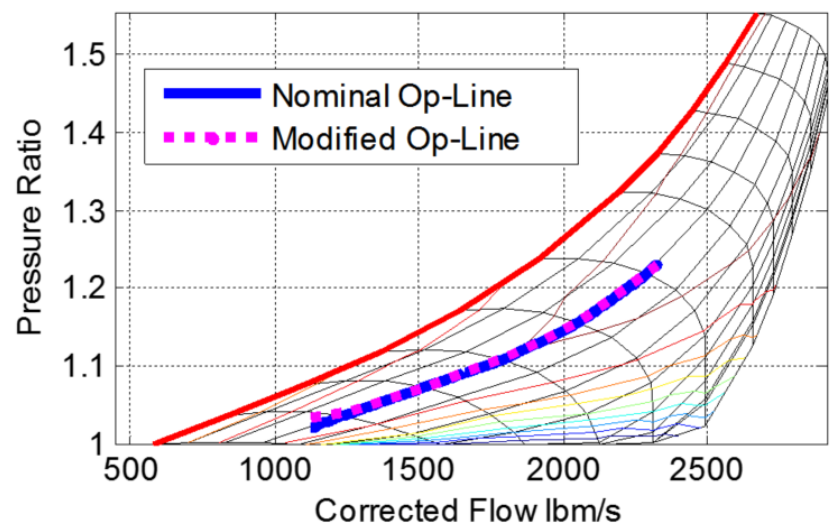

Figure 11. The fan op-line, showing how it is affected by the modifications to the VAFN schedule at low power.

The effect of the area change on transient performance was tested by running the same open-loop Wf acceleration profile with both VAFN schedules. Running with the modified schedule causes a reduction in minimum fan surge margin of $0.2 \%$ compared to the nominal schedule but eliminates issues with going off of the fan map during accelerations. The VAFN area at the higher power levels remained the same for both schedules, and thus the VAFN area during decelerations is almost the same with both schedules. Other changes observed when switching to the modified schedule include a reduction in max $T 40$ of $40^{\circ} \mathrm{R}$, a reduction in maximum $\mathrm{Nl}$ of $500 \mathrm{rpm}$, and a reduction in response time (an improvement) of 0.5 seconds. These results suggest that with the exception of a slight reduction in fan surge margin, the changed schedule generally improves performance and operability margins. Given this conclusion, the modified schedule was used for the remainder of this work.

\section{Dynamic Systems Analysis}

With a satisfactory baseline closed-loop controller design, control parameters can be varied to assess tradeoffs between various design quantities, mainly between dynamic performance and operability margins. Specifically, analysis of the relationship between the ability to meet the FAA 5-second requirement and the remaining, or minimum, surge margin during transients may identify possible design improvements.

To characterize the tradeoff between performance and operability for this $\mathrm{N}+3 \mathrm{GTF}$ engine, the $W f / P s 3$-based acceleration limiter was designed to protect 8 different HPC surge margin limits. The acceleration response time versus minimum HPC surge margin trend is

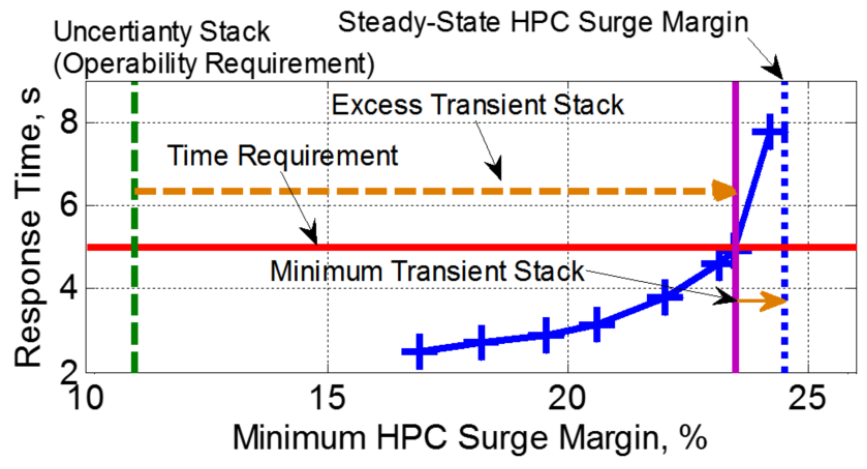

Figure 12. Response time versus minimum HPC surge margin achieved with Wf/Ps3 acceleration limiters tuned to protect eight different HPC surge margin targets. plotted in Figure 12, with blue crosses representing each of the 8 controller designs.

Observing this figure, the acceleration limiter solution that just meets the response time requirement has a minimum surge margin close to $23 \%$ (indicated with the vertical, solid purple line in Figure 12). Given that the minimum steady-state HPC surge margin is about $24.3 \%$ (vertical dotted blue line), the minimum transient stack needed to just meet the time requirement is about $1.3 \%$ (thin, solid orange arrow in the bottom right of the figure). Further, given a value for the uncertainty stack of $11 \%$ (vertical, green dashed line), the excess amount of transient stack in this engine design is, at most, $12 \%$ (dashed orange arrow). Note that since the steady-state HPC surge margin is $24.3 \%$, and the uncertainty stack is assumed to be $11 \%$, the size of the transient stack built into the engine design can be computed as the difference of the two, or $13.3 \%$.

Note that this analysis was performed using the nominal engine, and the effects of engine health parameter and other uncertainties were not captured. Further, this analysis was performed at SLS, standard day temperature, and does

American Institute of Aeronautics and Astronautics 
not capture the effect of differing ambient conditions. This means this study will give an approximate, optimistic figure for how much transient stack is needed. Therefore, instead of a value of 1.3\%, a revised transient stack of 5\% should provide this kind of engine plenty of margin for transients, given an appropriate controller design, and will still reduce the transient stack by more than half. The recommendation based on a transient stack of 5\% would be to consider engine design solutions with a steady-state HPC surge margin of as low as $16 \%$ (the uncertainty stack value of $11 \%$, plus the revised transient stack value of 5\%). If the HPC surge margin constraint was a limiting factor for fuel efficiency in this design, it is likely that better engine design solutions may be found if the design process is performed again with the revised $16 \%$ steady-state HPC surge margin constraint.

\section{Conclusions}

The study described in this work shows that the NASA geared turbofan (GTF) with the variable area fan nozzle (VAFN) concept is theoretically feasible, but the concept and especially its shape memory alloy (SMA) actuator present novel challenges. This work shows that, during rapid accelerations, the proposed area range and slow dynamics of the SMA-based VAFN actuator both have a significant impact on system performance and operability. This study shows that trying to accelerate the engine quickly, from idle to takeoff power, was problematic and caused the fan component to operate in or near the choked region. Reducing the fan nozzle area near idle power, from 16500 to 9000 sq. in, allowed the engine to accelerate and also maintain the desired fuel burn rates achieved at cruise. The dynamic analysis carried out indicates that the engine has significantly more high pressure compressor (HPC) surge margin (as much as $12 \%$ excess) than is necessary to meet the $5 \mathrm{~s}$ acceleration response time requirement for takeoff. This suggests that the engine's steady-state HPC surge margin constraint used for the engine design could be reduced, which potentially can result in significant efficiency improvements.

\section{Acknowledgements}

Thanks go to William Haller of NASA Glenn Research Center for his help guiding this work, and Thomas Lavelle and Scott Jones of NASA Glenn for their assistance with engine modeling and insight into the systems analysis process. The authors thank Sanjay Garg of NASA Glenn for his suggestions on improving the analysis of actuator dynamics in this paper, as well other colleague peer reviewers. Finally, the authors thank the NASA Advanced Air Transportation Technologies project for funding this work.

\section{References}

${ }^{1}$ Ashcraft, S.W., Padron, A.S., Pascioni, K.A, Stout Jr., G.W., and Huff, D.L., "Review of Propulsion Technologies for N+3 Subsonic Vehicle Concepts," NASA TM 2011-217239, October, 2011.

${ }^{2}$ Hathaway, M.D., Del Rosario, R., and Madavan, N.K., "NASA Fixed Wing Project Propulsion Research and Technology Development Activities to Reduce Thrust Specific Energy Consumption," NASA TM 2013-216548, July 2013.

${ }^{3}$ Antcliff, K.R., Guynn, M.D., Marien, T.V., Wells, D.P.., Schneider, S.J., and Tong, M.T., "Mission Analysis and Aircraft Sizing of Hybrid-Electric Regional Aircraft," AIAA 2016-1028, AIAA SciTech, 54 ${ }^{\text {th }}$ AIAA Aerospace Sciences Meeting, San Diego, CA, January 4-8, 2016.

${ }^{4}$ Felder, J.L., Brown, G.V, Dae Kim, H., and Chu, J., "Turboelectric Distributed Propulsion in a Hybrid Wing Body Aircraft," ISAB 2011-1340, $20^{\text {th }}$ International Society for Airbreathing Engines, Gothenburg, Sweden, September 1216, 2011.

${ }^{5}$ Welstead, J.R., and Felder, J.L., "Concept Design of a Single-Aisle Turboelectric Commercial Transport with Fuselage Boundary Layer Ingestion," AIAA 2016-1027, AIAA SciTech, $54^{\text {th }}$ AIAA Aerospace Sciences Meeting, San Diego, CA, January 4-8, 2016.

${ }^{6}$ Guynn, M.A., Berton, J.J., Tong, M.T., and Haller, W.J., "Advanced Single-Aisle Transport Propulsion Design Options Revistited," AIAA 2013-4330, AIAA Aviation, 2013 Aviation Technology, Integration, and Operations Conference, Los Angeles, CA, August 12-14, 2013.

7 Mabe, J., "Variable Area Jet Nozzle for Noise Reduction Using Shape Memory Alloy Actuators," European Acoustics Association, Acoustics'08 Paris, pp 5487-5492, Paris, France, June 29-July4, 2008.

${ }^{8}$ Michel, U., "The Benefits of Variable Area Fan Nozzles on Turbofan Engines," AIAA 201-226, 49 ${ }^{\text {th }}$ AIAA Aerospace Sciences Meeting including the New Horizons Forum and Aerospace Exposition," Orlando, FL, January 4-7, 2011.

${ }^{9}$ United Technologies/Pratt and Whitney, The Aircraft Gas Turbine Engine and its Operation, Instruction Manual, P\&W Part No. 182408, December 1982.

American Institute of Aeronautics and Astronautics 
${ }^{10}$ Song, G., Ma, N., Lee, H.-J., and Arnold, S., "Design and Control of a Proof-of-Concept Variable Area Exhaust Nozzle Using Shape-Memory Alloy Actuators," IOP Publishing, Smart Materials and Structures, Vol 16, pp 13421347, June 2007.

${ }^{11}$ Majima, S., Kodama, K., and Hasegawa, T., "Modeling of Shape Memory Alloy Actuator and Tracking Control System with the Model." Control Systems Technology, IEEE Transactions on Control Systems Technology, Volume 9, Issue 1, Pages 54-59, January 2001.

${ }^{12}$ Claus, R.W., Evans, A.L., Lylte, J.K. and Nichols, L.D., "Numerical Propulsion System Simulation," Computing Systems in Engineering, Vol 2, no 4, pp 357-364, 1991.

${ }^{13}$ Chin, J.C., Csank, J.T., Haller, W.J., and Seidel, J.A., "An Introduction to Transient Engine Applications Using the Numerical Propulsion System Simulation (NPSS) and MATLAB," NASA, NASA/TM 2016-218922, January, 2106.

${ }^{14}$ Kurtzke, J., "Correlations Hidden in Compressor Maps" GT2011-45519, ASME Turbo Expo 2011, Vancouver, BC, June 6-10, 2011.

${ }^{15}$ Volponi, A.J., "Gas Turbine Parameter Corrections," Journal of Engineering for Gas Turbines and Power, Vol. 121 Issue 4, October 1999

${ }^{16}$ Csank, J.T., and Zinnecker, A.M., "Application of the Tool for Turbine Engine Closed-loop Transient Analysis (TTECTrA) for Dynamic Systems Analysis," AIAA 2014-3975, 50 ${ }^{\text {th }}$ AIAA/ASME/SAE/ASEE Joint Propulsion Conference, Cleveland, OH, July 28-30, 2014.

${ }^{17}$ Zinnecker, A.M., and Csank, J.T., "A Methodology to Assess the Capability of Engine Designs to Meet Closedloop Performance and Operability Requirements," AIAA 2015-4023, 51 ${ }^{\text {st }}$ AIAA/SAE/ASEE Joint Propulsion Conference, Orlando, FL, July 27-29, 2015.

${ }^{18}$ Walsh, P. P., and Fletcher, P., "Gas Turbine Performance," Blackwell Science Ltd and ASME Press, 2004.

${ }^{19}$ Federal Aviation Administration, "Title 14 of the Code of Federal Regulations", http://www.ecfr.gov/cgi-bin/textidx?SID=aa3a99819fbf162f142eee0ec759f8df\&mc=true\&node=se14.1.33 173\&rgn=div8, accessed April, 2016.

${ }^{20}$ Csank, J.T., and Zinnecker, A.M., "Tool for Turbine Engine Closed-Loop Transient Analysis (TTECTrA) Users' Guide,” NASA TM/2014-216663, November, 2014.

${ }^{21}$ Chin, J.C., and Csank, J.T., "Tool for the Integrated Dynamic Numerical Propulsion System Simulation (NPSS)/Turbine Engine Closed-Loop Transient Analysis (TTECTrA) User's Guide," NASA, NASA/TM 2016218923.

${ }^{22}$ Csank, J.T., May, R.D., Litt, J.S., and Guo, T.-H., "Control Design for a Generic Commercial Aircraft Engine," AIAA 2010-6629, $46^{\text {th }}$ AIAA/ASME/SAE/ASEE Joint Propulsion Conference and Exhibit, Nashville, TN, July 25$28,2010$.

${ }^{23}$ Behbahani A., Culley D., Carpenter S., Mailander B., Hegwood B., Smith B., Darouse C., Mahoney T., Quinn R., Battestin G., Roney W. "Status, Vision, and Challenges of an Intelligent Distributed Engine Control Architecture." SAE Technical Paper; 2007 Sep 17.

${ }^{24}$ Connolly, J.W., Csank, J.T., Chicatelli, A., and Kilver, J., "Model-Based Control of a Nonlinear Aircraft Engine Simulation using an Optimal Tuner Kalman Filter Approach," AIAA 2013-4002, $49^{\text {th }}$ AIAA/ASME/SAE/ASEE Joint Propulsion Conference, San Jose, CA, July 14-17, 2013.

${ }^{25}$ Csank, J.T, and Connolly, J.W., "Model-Based Engine Control Architecture with an Extended Kalman Filter," AIAA 2016-1623, AIAA SciTech, AIAA Guidance, Navigation, and Control Conference, San Diego, CA, January 4$8,2016$.

American Institute of Aeronautics and Astronautics 